\title{
A Novel Output Prediction Method in Production Management Based on Parameter Evaluation Using DHNN
}

\author{
Jiantao Chang, Yuanying Qiu, and Xianguang Kong \\ Key Laboratory of Electronic Equipment Structure Design (Xidian University), Ministry of Education, Xian 710071, China \\ Correspondence should be addressed to Jiantao Chang; taocj81@163.com
}

Received 29 July 2013; Revised 11 November 2013; Accepted 12 November 2013

Academic Editor: Yuri Sotskov

Copyright (C) 2013 Jiantao Chang et al. This is an open access article distributed under the Creative Commons Attribution License, which permits unrestricted use, distribution, and reproduction in any medium, provided the original work is properly cited.

\begin{abstract}
Output prediction is one of the difficult issues in production management. To overcome this difficulty, a dynamic-improved multiple linear regression model based on parameter evaluation using discrete Hopfield neural networks (DHNN) is presented. First, a traditional multiple linear regression model is established; this model takes the factors in production lifecycle (not only one phase of the production) into account, such as manufacturing resources, manufacturing process, and product rejection rate, so it makes the output prediction be more accurate. Then a static-improved model is built using the backstepping method. Finally, we obtain the dynamic-improved model based on parameter evaluation using DHNN. These three models are applied to an aviation manufacturing enterprise based on the actual data, and the results of the output prediction show that the models have practical value.
\end{abstract}

\section{Introduction}

Production management is the general term for the management of the production system. Production management mainly contains two aspects, the job of production planning and production control [1]. The job of production planning contains production planning design, technical preparation planning, and production scheduling. The job of production control contains production progress control, production inventory control, production quality control, and production cost control. Figure 1 gives the function structure of production management.

Output prediction is one of the key technologies in production planning and production control. If the output prediction is inaccurate, the difficulty of production planning design, the production cost control, and inventory control will appear. And then the production efficiency will become low and the waste of human and material resources will become high.

Many contributions to production management and output prediction have been made in the last two decades from various perspectives. Those studies can be classified into three groups: ERP (enterprise resources planning), MES (manufacturing execution system), and output prediction.
Hung et al. [2] provided a broader, richer model of knowledge transfer networks to promote insight into successful ERP implementation. In practice, the key to effective knowledge transfer is the establishment of a positive knowledge transfer climate. To achieve a successful ERR implementation, practitioners should focus on developing a positive relationship with ERR implementation partners. The purpose of this paper was to examine the impact of the knowledge transfer climate and relationship bonding. Ho and Ireland [3] examined the effect of forecasting errors in the context of an ERP-controlled manufacturing system. Then considered a mitigating remedy, the use of a lot-sizing rule, to cope with the consequences of forecasting inaccuracy without resorting to costly inventoryoriented buffers. An ERP-controlled manufacturing system was simulated to see how these lot-sizing rules mitigate the forecast errors and subsequently generate acceptable system performance. Koh et al. [4] proposed a knowledge management approach to tackle uncertainty in ERP-controlled manufacturing environment. The development of a knowledgebased ERP planning model and a knowledge-based ERPcontrolled manufacturing simulation model within which knowledge of tackling underlying causes of uncertainty can be stored for future reference is discussed. A combination of explicit and tacit knowledge was created and applied 


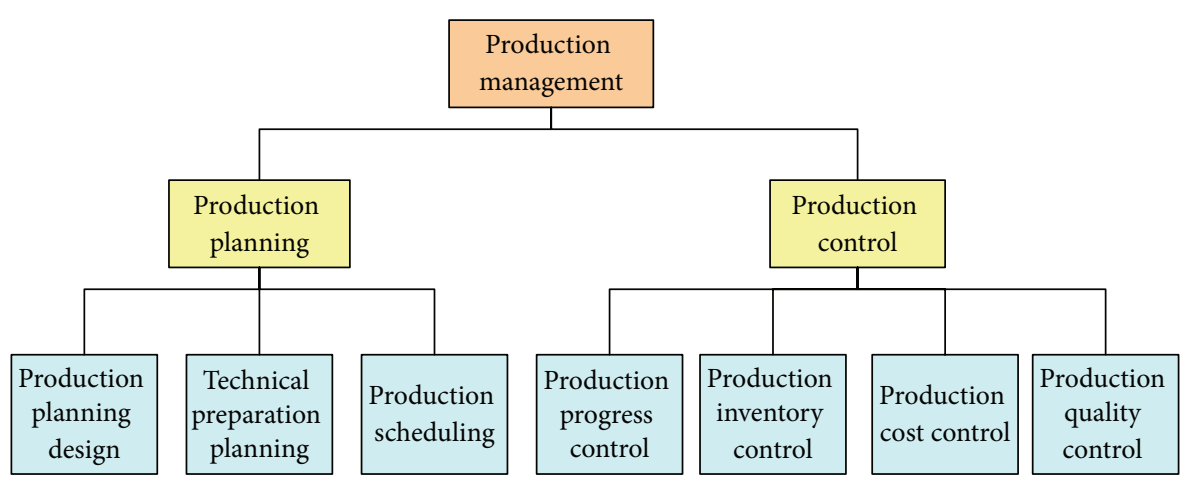

FIGURE 1: Function structure of production management.

to update the planned parameters and to decide for the appropriate buffering or dampening techniques to tackle the underlying causes of uncertainty. Matsveichuk et al. [5] addressed the issue of how to best execute the schedule in a two-phase scheduling decision framework by considering a two-machine flow-shop scheduling problem in which each uncertain processing time of a job on a machine may take any value between a lower and upper bound. The scheduling objective was to minimize the makespan. The approach of this paper enabled a scheduler to best execute a schedule and may end up with executing the schedule optimally in many instances according to our extensive computational experiments. The algorithm for testing the set of sufficient conditions of schedule domination is not only theoretically appealing but also empirically fast. They also addressed the flow-shop minimum-length scheduling problem with $n$ jobs processed on two machines. The authors investigated properties of this partial job order and showed how to construct this order in polynomial time. The approach based on a set of dominant schedules allowed the authors to find special cases of the problem when it was possible to find an optimal schedule in spite of the uncertainty of the numerical data [6]. Matsveichuk et al. [7] addressed the flow-shop minimum-length scheduling problem with $n$ jobs processed on two machines where processing times are uncertain. The authors obtained necessary and sufficient conditions for the case when it is possible to fix the order of two jobs in a minimal set of dominant schedules. The necessary and sufficient conditions were proven for the case when one schedule dominates all the others. They characterized also the case where there does not exist nontrivial schedule domination. Zhong et al. [8] presented an RFID-enabled real-time manufacturing execution system (RT-MES). RFID devices were deployed systematically on the shop floor to track and trace manufacturing objects and collect real-time production data. The efficiency and effectiveness of the proposed RTMES were evaluated with real-life industrial data for shopfloor production management in terms of workers, machines, and materials. Huin et al. [9] presented the various strategic and operational requirements of regional M-SMES and proposed a knowledge-based resources planning model. This improved AI model made use of the large amount of accumulated knowledge typically found in the M-SMES, especially those in the electronics and precision engineering sectors. Amirteimoori and Kordrostami [10] introduced a data envelopment analysis approach to making future production plans in a centralized decision-making environment when demand changes can be forecasted in the next production season. The approach proposed took the size of operational units into account so that the planned production for each unit becomes proportionate to the ability of the units, and a DEA-based production planning approach was developed to determine the most favorable production plans. Golany [11] presented an interactive linear programming procedure to set up goals for desired outputs. Their procedure is based on the empirical production functions generated by DEA and then adjusted by new information provided by the decision maker in each iteration. Du et al. [12] proposed two planning ideas. One is optimizing the average or overall production performance of the entire organization, measured by the CCR (Cooper and Rhodes) efficiency of the average input and output levels of all units. The other is simultaneously maximizing total outputs produced and minimizing total inputs consumed by all units. Korhonen and Syrjänen [13] developed an interactive approach based on DEA and multiple-objective linear programming technique to a resource-allocation problem that typically appears in a centralized decision-making environment. By simultaneously maximizing the total amount of output variables produced by each individual unit, an efficient resource-allocation solution as well as all the output amounts can be obtained. Kim et al. [14] presented a new integrated model for production planning and scheduling for multi-item and multilevel production. Then he in addition presented a mathematical formulation, proposed a heuristic solution procedure, and demonstrated the performance of the model by comparing the experimental results with those of a traditional approach and optimal solution. Lasserre [15] and Dauzère-Péres and Lasserre [16] addressed the integrated lot-sizing and scheduling problem and proposed a two-level, iterative procedure to compute the best possible schedule in a job-shop environment. Anwar and Nagi [17] proposed a two-phase heuristic for a general job shop that produces 
complex assemblies. In the first phase, the proposed heuristic schedules operate by exploiting the critical path to minimize the makespan on a lot-for-lot basis. In the second phase, the heuristic iterative groups order to determine lot sizes that further reduce the makespan, setup, and holding cost. Xiong and Nyberg [18] discussed production plan and scheduling system of refinery CIMS, where production procedure process, basic production characters, refinery production and administration, and its activity types are introduced; then a refinery integrated production plan and schedule system is put forward. Zhang and Yan [19] addressed an integrated job-shop production planning and scheduling problem with setup time and batches. An improved hybrid genetic algorithm (HGA) is given in order to simultaneously optimize the production plan and the schedule. At last a comparison is made between the hybrid algorithm and a hierarchical production planning and scheduling method. Fandel and Stammen-Hegene [20] developed a model for multiproduct and multilevel production; they could solve only small-sized problems with a small number of products and macroperiods because of their model's complexity. Chazal et al. [21] studied the production planning and inventory management problem based on the assumption that the firm under consideration performs in continuous time on a finite period in order to dynamically maximize its instantaneous profit. Pochet and Wolsey [22] operated the linear programming-based production planning tools typically at a higher, aggregate production level. In order to address production planning problems at a deeper detail, integer and, often, binary variables need to be introduced into the mathematical model and this led to more complex models such as the ones described. Damodaran and Vélez-Gallego [23] presented a simulated annealing algorithm to minimize the makespan on a group of identical batch processing machines arranged in parallel, the case where each job had an arbitrary processing time, nonidentical size, and nonzero ready time which was considered.

Although great progress has been made in the studies on production management, most of the studies focused on shop scheduling problems, so little research developed output prediction approaches. These approaches also have limitations due to their range of data extraction as these approaches obtained parameter only at a certain phase but not in the lifecycle of production. Furthermore, little research used dynamic-improved multiple linear regression model to predict the output.

In order to overcome these shortcomings above, this paper focused on a dynamic-improved multiple linear regression model based on DHNN to predict the output. The parameters of this mathematical model will contain the factors in production management lifecycle. So the output prediction will be more accurate and reasonable.

The rest of this paper is organized as follows. The following section discusses the DHNN evaluation method. Section 3 explains the mathematical models to predict output. Section 4 constructs three practical mathematical models, and Section 5 evaluates the models and analyzes the error to test the hypotheses in an aviation manufacturing enterprise. Finally, Section 6 presents conclusions.

\section{DHNN Evaluation Method}

DHNN is a one-layer neural network, and its input variables and output variables are Boolean type. Figure 2 illustrates the structure of DHNN. The input of the network is $I_{1}, I_{2}, \ldots, I_{n}$, and the output is $V_{1}, V_{2}, \ldots, V_{n}$. The relationship of the input and output is as follows [24]:

$$
\begin{gathered}
x_{j}(t)=\sum w_{i j} V_{j}+I_{j}, \quad j=1,2, \ldots, n, \\
V_{i}(t+1)=\operatorname{sgn}(x j(t)), \quad i, j=1,2, \ldots, n .
\end{gathered}
$$

In (1), $\operatorname{sgn}(*)$ is a symmetric step function, and its output is +1 or $-1 . W=\left[w_{i j}\right]_{n \times n}$ is the connection weight matrix of network and $w_{i j}$ represents the connection weight of the neurons from $i$ to $j$.

DHNN is mainly used to associative memory and the output $V$ can be obtained through feedback evolution of the network when the input is $I . V$ is a stable memory from the evolution of $I$.

The process of DHNN evaluation can be divided into memory process and association process. In order to realize the DHNN evaluation, we can use the steps as follows.

Step 1. Based on the samples $V^{1}, V^{2}, \ldots, V^{n}$ which required memory, calculate the weight by (2) as follows:

$$
w_{i j}= \begin{cases}\sum_{k=1}^{m} V_{i}^{k} \mid V_{j}^{k}, & i \neq j \\ 0, & i=j,\end{cases}
$$

where $I_{j}=0$ and $I_{j}$ is the initial input of the network.

Step 2. Let the test sample be the initial value of the network output, and let $V^{\tau}$ be an arbitrary input vector; then

$$
V\left(t_{0}\right)=V^{\tau}, \quad V \in R^{n} .
$$

Step 3. Calculate the following iterative formula:

$$
V_{i}(t+1)=\operatorname{sgn}\left(\sum_{j=1}^{n} w_{i j} V_{j}(t)\right) .
$$

Step 4. Repeat iterations, until each unit becomes unchanged

$$
V_{i}(t+1)=V_{j}(t) .
$$

Thus, $V^{\tau}$ will be back to a memory sample.

The allocation of evaluation weight can be obtained through the method of neuron reasonable grouping; neurons in one group have the same evaluation, and, if the evaluation has a large weight, the number of neurons in this group will be large; then the impact of evaluation result for the network will be big.

Let the number of evaluation be $m$, the weight of these evaluation $w_{i}$, and $1<i<m$; then $0<w_{i}<1$ and $\sum w_{i}=1$. Let the group of $w_{i}$ be $G_{i}$ and the number of neuron $n_{i}$ as follows:

$$
n_{i}=\operatorname{RND}\left(100 \times w_{i}\right) .
$$


In (6), $\mathrm{RND}(*)$ is a rounding function and the sum of $n_{i}$ must be 100 . Hence, the effect of weight will be included into the evaluation result. The sum of neurons in each level is as follows:

$$
N=\sum_{i}^{m} n_{i}
$$

The memory capacity of DHNN is $(0.13 \sim 0.15) \times N$. If the sample of memory mode is smaller than 13 , the DHNN designed by the method above will be reasonable.

\section{Mathematical Models for Production Prediction}

To predict the output of the production plan accurately is the precondition of production management. We can use a variety of mathematical models to predict the output, such as traditional multiple linear regression model, static improved multiple linear regression model, and dynamic improved multiple linear regression model.

The traditional multiple regression model which includes $n$ sets of observation values can be given as follows:

$$
\begin{aligned}
y_{1}= & \beta_{0}+\beta_{1} x_{11}+\beta_{2} x_{12}+\cdots+\beta_{p} x_{1 p}, \\
y_{2}= & \beta_{0}+\beta_{1} x_{21}+\beta_{2} x_{22}+\cdots+\beta_{p} x_{2 p}, \\
& \vdots \\
y_{n}= & \beta_{0}+\beta_{1} x_{n 1}+\beta_{2} x_{n 2}+\cdots+\beta_{p} x_{n p} .
\end{aligned}
$$

Written in matrix form,

$$
Y=X \beta,
$$

where

$$
\begin{aligned}
Y=\left[\begin{array}{c}
y_{1} \\
y_{2} \\
y_{3} \\
\vdots \\
y_{n}
\end{array}\right], \quad X & =\left[\begin{array}{ccccc}
1 & x_{11} & x_{12} & \cdots & x_{1 p} \\
1 & x_{21} & x_{22} & \cdots & x_{2 p} \\
1 & x_{31} & x_{32} & \cdots & x_{3 p} \\
\vdots & \vdots & \vdots & \ddots & \vdots \\
1 & x_{n 1} & x_{n 2} & \cdots & x_{n p}
\end{array}\right], \\
\beta & =\left[\begin{array}{c}
\beta_{0} \\
\beta_{1} \\
\beta_{1} \\
\vdots \\
\beta_{p}
\end{array}\right] .
\end{aligned}
$$

$\beta$ is the regression coefficient. We can use the least squares method to estimate the value. The formula can be given as follows:

$$
Q=\sum_{i=1}^{n} e_{i}^{2}=e^{\prime} e=\left(y-x^{\beta}\right)^{\prime}\left(y-x^{\beta}\right),
$$

where $e_{i}=y_{i}-\widehat{y}_{i}$, and $e=\left(e_{1}, e_{2}, \ldots, e_{n}\right)^{\prime}$.
We can obtain two kinds of improved multiple linear regression model, the static-improved model and the dynamic-improved one.

In order to get the static-improved model, first, we should get the OLSE (ordinary least square estimation). $\widehat{\beta_{0}}, \widehat{\beta_{1}}, \ldots, \widehat{\beta_{p}}$ is the estimate value of $\beta_{0}, \beta_{1}, \ldots, \beta_{p}$, and its squared deviations is as follows:

$$
\begin{aligned}
Q & \left(\beta_{0}, \beta_{1}, \ldots, \beta_{p}\right) \\
& =\sum_{i=1}^{n}\left(y_{i}-\beta_{0}-\beta_{1} x_{i 1}-\beta_{2} x_{i 2}-\cdots-\beta_{p} x_{i p}\right)^{2} .
\end{aligned}
$$

We should get the minimum of 1 squared deviation as follows:

$$
\begin{aligned}
Q & \left(\widehat{\beta_{0}}, \widehat{\beta_{1}}, \ldots, \widehat{\beta_{p}}\right) \\
& =\sum_{i=1}^{n}\left(y_{i}-\widehat{\beta_{0}}-\widehat{\beta_{1}} x_{i 1}-\widehat{\beta_{2}} x_{i 2}-\cdots-\widehat{\beta_{p}} x_{i p}\right)^{2} \\
& =\min _{\beta_{0}, \beta_{1}, \ldots, \beta_{p}} \sum_{i=1}^{n}\left(y_{i}-\beta_{0}-\beta_{1} x_{i 1}-\beta_{2} x_{i 2}-\cdots-\beta_{p} x_{i p}\right)^{2} .
\end{aligned}
$$

$\widehat{\beta_{0}}, \widehat{\beta_{1}}, \ldots, \widehat{\beta_{p}}$ should satisfy the following equation group:

$$
\begin{aligned}
& \left.\frac{\partial Q}{\partial \beta_{0}}\right|_{\beta_{0}-\widehat{\beta_{0}}} \\
& \quad=-2 \sum_{i=1}^{n}\left(y_{i}-\widehat{\beta_{0}}-\widehat{\beta_{1}} x_{i 1}-\widehat{\beta_{2}} x_{i 2}-\cdots-\widehat{\beta_{p}} x_{i p}\right)^{2}=0,
\end{aligned}
$$

$$
\begin{aligned}
& \left.\frac{\partial Q}{\partial \beta_{1}}\right|_{\beta_{1}-\widehat{\beta_{1}}} \\
& \quad=-2 \sum_{i=1}^{n}\left(y_{i}-\widehat{\beta_{0}}-\widehat{\beta_{1}} x_{i 1}-\widehat{\beta_{2}} x_{i 2}-\cdots-\widehat{\beta_{p}} x_{i p}\right)^{2} x_{i 1}=0,
\end{aligned}
$$

$$
\begin{aligned}
& \left.\frac{\partial Q}{\partial \beta_{0}}\right|_{\beta_{p}-\widehat{\beta_{p}}} \\
& \quad=-2 \sum_{i=1}^{n}\left(y_{i}-\widehat{\beta_{0}}-\widehat{\beta_{1}} x_{i 1}-\widehat{\beta_{2}} x_{i 2}-\cdots-\widehat{\beta_{p}} x_{i p}\right)^{2} x_{i p}=0 .
\end{aligned}
$$

If $\left(X^{\prime} X\right)^{-1}$ exists, then the OLSE is

$$
\widehat{\beta}=\left(x^{\prime} x\right)^{-1} x^{\prime} y .
$$


So we can get 4 parameters which can reflect the effect of the regression. These 4 parameters are $q, u, F, r$, and $t_{j}$. The formulas can be given as follows:

$$
\begin{gathered}
u=\sum_{i=1}^{n}\left(\hat{y}_{i}-\bar{y}\right)^{2}, \\
F=\frac{u / p}{q /(n-p-1)}, \\
r=\sqrt{\frac{u}{(u+p)}}, \\
t_{j}=\frac{\widehat{\beta}_{j}}{\sqrt{c_{i j}[q /(n-p-1)]}},
\end{gathered}
$$

where $c_{i j}=\left(x^{\prime} x\right)^{-1}, i, j=0,1,2, \ldots, p$.

$t_{j}$ can be used to test the significance of the regression coefficients. We use the backstepping method of $t_{j}$ to remove the low significance variables, because if, $t_{j}$ is small, the effect to $Y$ from $X$ is not obvious. All the variables will be tested by $t_{j}$ in proper order until the variables left can meet the requirements of the significant level $\alpha>0.05$. And then the static-improved multiple linear regression model is obtained.

On the other hand, in order to obtain the dynamicimproved model, the low weight independent variables can be removed by the weight analysis method based on DHNN evaluation, and then the dynamic-improved multiple linear regression model will be established.

\section{Application and Analysis of the Mathematical Models}

The impact factors of output prediction include several aspects, for example, production conditions, labor input, and some factors in production process. In production conditions aspect, sum of equipment is an important parameter; in labor input aspect, labor hours are an important parameter. The factors in production process also play an important role, for example, rejection rate. So the 7 parameters are total number of equipment $\left(x_{1}\right)$, number of running equipment $\left(x_{2}\right)$, number of added equipment $\left(x_{3}\right)$, labor hours last season $\left(x_{4}\right)$, rejection rate $\left(x_{5}\right)$, average number of processes $\left(x_{6}\right)$, and output last season $\left(x_{7}\right)$.

4.1. Traditional Model. We obtain the 7 parameters of an aviation manufacturing enterprise from 1996 to 2012 as shown in Table 1.

The top 11 lines of data (1996-2007) in Table 1 are used to determine the parameters of the model. The remaining 5 lines of data (2008-2012) are used to verify the accuracy and practicality of the mathematical models.

We establish the traditional multiple linear regression equation as follows:

$$
y=\beta_{0}+\beta_{1} x_{1}+\beta_{2} x_{2}+\beta_{3} x_{3}+\beta_{4} x_{4}+\beta_{5} x_{5}+\beta_{6} x_{6}+\beta_{7} x_{7} .
$$

By applying the proposed equation (20), average regression coefficients can be calculated using the top 11 lines of data in Table 1. Then we obtain the rolling approach model (Model A) based on the data in 1997-2007, 1998-2008, 1999-2009, 20002010, and 2001-2011 as follows:

$$
\begin{aligned}
y_{2007}= & -10.6522-0.0258 x_{1}+0.0315 x_{2}+0.0018 x_{3} \\
& +0.1024 x_{4}+19.3016 x_{5}+0.1742 x_{6}+0.8203 x_{7}, \\
y_{2008}= & -10.4604-0.0335 x_{1}+0.0275 x_{2}+0.0019 x_{3} \\
& +0.1018 x_{4}+19.2036 x_{5}+0.1831 x_{6}+0.7985 x_{7}, \\
y_{2009}= & -10.5823-0.0342 x_{1}+0.0323 x_{2}+0.0022 x_{3} \\
& +0.1045 x_{4}+19.8013 x_{5}+0.1861 x_{6}+0.7704 x_{7}, \\
y_{2010}= & -10.76-0.0350 x_{1}+0.0280 x_{2}+0.0015 x_{3} \\
& +0.1009 x_{4}+19.4018 x_{5}+0.1921 x_{6}+0.8315 x_{7}, \\
y_{2011}= & -10.3875-0.0312 x_{1}+0.0302 x_{2}+0.0016 x_{3} \\
& +0.1029 x_{4}+19.2066 x_{5}+0.1951 x_{6}+0.8428 x_{7} .
\end{aligned}
$$

4.2. Static-Improved Model. We can obtain the staticimproved model by significance test of the regression coefficients. The measurement of parameters selection is $\alpha$; if $\alpha<0.05$, the parameter will be removed. Table 2 gives the process of the selection. For example, in the first round, the $t$ value 0.0995 is calculated by (19), and the significant weight $\alpha<0.05$; then by (17), we can obtain $F<F_{\alpha}$, so number of added equipment is removed. After 4 rounds of selection, the removed parameters are number of added equipment, rejection rate, and average number of parts processes.

So we can determine the important factors affecting output prediction in this aeronautical enterprise as follows:

(i) total number of equipment;

(ii) number of running equipment;

(iii) labor hours last season;

(iv) output last season.

The static-improved multiple linear regression model (Model B) which is obtained by backstepping method can be expressed as

$$
\begin{aligned}
y_{2007}= & -10.6522-0.0258 x_{1}+0.0315 x_{2}+0.1024 x_{4} \\
& +0.8203 x_{7} \\
y_{2008}= & -10.4604-0.0335 x_{1}+0.0275 x_{2}+0.1018 x_{4} \\
& +0.7985 x_{7}
\end{aligned}
$$


TABLE 1: Basic datum which affects output prediction in an aviation manufacturing enterprise.

\begin{tabular}{|c|c|c|c|c|c|c|c|c|}
\hline Season & $\begin{array}{l}\text { Total number } \\
\text { of equipment }\end{array}$ & $\begin{array}{l}\text { Number of } \\
\text { running } \\
\text { equipment }\end{array}$ & $\begin{array}{l}\text { Number of } \\
\text { added } \\
\text { equipment }\end{array}$ & $\begin{array}{l}\text { Labor hours } \\
\text { last season }\end{array}$ & $\begin{array}{l}\text { Rejection } \\
\text { rate }\end{array}$ & $\begin{array}{c}\text { Average number of } \\
\text { parts process }\end{array}$ & $\begin{array}{l}\text { Output last } \\
\text { season }\end{array}$ & $\begin{array}{l}\text { Output this } \\
\text { season }\end{array}$ \\
\hline 1996 & 704 & 587 & 236 & 200.68 & 0.69 & 5.07 & 27 & 33 \\
\hline 1997 & 790 & 634 & 253 & 246.87 & 0.63 & 5.44 & 33 & 36 \\
\hline 1998 & 880 & 707 & 352 & 289.22 & 0.59 & 5.77 & 36 & 43 \\
\hline 1999 & 1030 & 858 & 344 & 329.33 & 0.56 & 6.04 & 43 & 50 \\
\hline 2000 & 1201 & 942 & 482 & 370.29 & 0.56 & 6.48 & 50 & 58 \\
\hline 2001 & 1329 & 959 & 536 & 394.42 & 0.6 & 6.79 & 58 & 62 \\
\hline 2002 & 1510 & 1000 & 501 & 436.39 & 0.6 & 7.38 & 62 & 67 \\
\hline 2003 & 1624 & 1057 & 522 & 468.77 & 0.54 & 7.3 & 67 & 70 \\
\hline 2004 & 1703 & 1086 & 427 & 525.35 & 0.51 & 7.44 & 70 & 73 \\
\hline 2005 & 1813 & 1125 & 343 & 558.68 & 0.48 & 7.7 & 73 & 77 \\
\hline 2006 & 1841 & 1165 & 379 & 550.63 & 0.44 & 7.91 & 77 & 78 \\
\hline 2007 & 1884 & 1234 & 445 & 602.78 & 0.46 & 8.23 & 78 & 86 \\
\hline 2008 & 2319 & 1841 & 521 & 642.24 & 0.42 & 8.61 & 86 & 101 \\
\hline 2009 & 2893 & 2601 & 590 & 652.68 & 0.42 & 8.87 & 101 & 119 \\
\hline 2010 & 3288 & 2754 & 659 & 845.5 & 0.45 & 8.86 & 119 & 135 \\
\hline 2011 & 3935 & 3174 & 973 & 1001.07 & 0.42 & 8.49 & 135 & 150 \\
\hline 2012 & 4104 & 3697 & 1173 & 1099.57 & 0.43 & 8.6 & 150 & 160 \\
\hline
\end{tabular}

TABLE 2: $t$ value in selection process.

\begin{tabular}{lcccccc}
\hline $\begin{array}{l}\text { Selection } \\
\text { rounds }\end{array}$ & $\begin{array}{c}\text { Total number of } \\
\text { equipment }\end{array}$ & $\begin{array}{c}\text { Number of } \\
\text { running } \\
\text { equipment }\end{array}$ & $\begin{array}{c}\text { Number of } \\
\text { added } \\
\text { equipment }\end{array}$ & $\begin{array}{c}\text { Labor hours } \\
\text { last season }\end{array}$ & $\begin{array}{c}\text { Rejection rate } \\
\text { of parts process } \\
\text { of perason }\end{array}$ \\
\hline 1 & 2.1671 & 1.0983 & 0.0995 & 1.6932 & 0.1529 & 0.0442 \\
2 & 2.238 & 1.4404 & & 1.9948 & 0.15 & 0.0605 \\
3 & 2.317 & 1.4967 & & 2.0958 & 0.1431 & 5.3104 \\
4 & 2.3691 & 1.9336 & & 2.1545 & & 7.5273 \\
\hline
\end{tabular}

TABLE 3: The relative importance ratio of evaluation parameters.

\begin{tabular}{cccccccc}
\hline & $x_{1}$ & $x_{2}$ & $x_{3}$ & $x_{4}$ & $x_{5}$ & $x_{6}$ \\
\hline$x_{1}$ & 1 & 5 & 5 & 9 & 3 & 3 & 1 \\
$x_{2}$ & $1 / 5$ & 1 & $1 / 2$ & 1 & 3 & $1 / 2$ & $1 / 3$ \\
$x_{3}$ & $1 / 5$ & 2 & $1 / 3$ & 1 & $1 / 5$ & $1 / 3$ \\
$x_{4}$ & $1 / 9$ & $1 / 3$ & 2 & 5 & 1 & $1 / 7$ \\
$x_{5}$ & $1 / 3$ & 1 & 3 & 7 & $1 / 2$ & 1 \\
$x_{6}$ & $1 / 3$ & 3 & 4 & 8 & $1 / 2$ & 1 \\
$x_{7}$ & $1 / 2$ & 4 & & $1 / 2$ & $1 / 2$ & 1 \\
\hline
\end{tabular}

TABLE 4: The weight of every evaluation parameter.

\begin{tabular}{lccccccc}
\hline Evaluation parameters & $x_{1}$ & $x_{2}$ & $x_{3}$ & $x_{4}$ & $x_{5}$ & $x_{6}$ & 0.18 \\
\hline Weight & 0.40 & 0.05 & 0.05 & 0.19 & 0.19 & 0.12 \\
\hline
\end{tabular}

TABLE 5: Models' scores of every evaluation parameter.

\begin{tabular}{lccccccc}
\hline \multirow{2}{*}{ Models } & & \multicolumn{3}{c}{ Evaluation parameters } & $x_{5}$ & $x_{6}$ \\
& $x_{1}$ & $x_{2}$ & $x_{3}$ & $x_{4}$ & 7 & 5 \\
Model A & 5 & 6 & 7 & 5 & 8 & 6 \\
Model B & 7 & 8 & 6 & 7 & 8 & 7 \\
Model B & 8 & 5 & 8 & 7 & 8 \\
\hline
\end{tabular}


TABLE 6: Output status of the neural network.

\begin{tabular}{lcccccccc}
\hline Level & $1-100$ & $101-200$ & $201-300$ & $301-400$ & $401-500$ & $501-600$ & $601-700$ & $701-800$ \\
\hline 1 & 1 & -1 & -1 & -1 & -1 & -1 & -1 & -1 \\
2 & 1 & 1 & -1 & -1 & -1 & -1 & -1 & -1 \\
3 & 1 & 1 & 1 & -1 & -1 & -1 & -1 & -1 \\
4 & 1 & 1 & 1 & 1 & -1 & -1 & -1 & -1 \\
5 & 1 & 1 & 1 & 1 & 1 & -1 & -1 & -1 \\
6 & 1 & 1 & 1 & 1 & 1 & 1 & -1 & -1 \\
7 & 1 & 1 & 1 & 1 & 1 & 1 & 1 & -1 \\
8 & 1 & 1 & 1 & 1 & 1 & 1 & 1 & 1 \\
\hline
\end{tabular}

$$
\begin{aligned}
y_{2009}= & -10.5823-0.0342 x_{1}+0.0323 x_{2}+0.1045 x_{4} \\
& +0.7704 x_{7}, \\
y_{2010}= & -10.76-0.0350 x_{1}+0.0280 x_{2}+0.1009 x_{4} \\
& +0.8315 x_{7}, \\
y_{2011}= & -10.3875-0.0312 x_{1}+0.0302 x_{2}+0.1029 x_{4} \\
& +0.8428 x_{7} .
\end{aligned}
$$

4.3. Dynamic-Improved Model. We can obtain the dynamicimproved model by the method of DHNN evaluation. In the DHNN evaluation we use total number of equipment $\left(x_{1}\right)$, number of running equipment $\left(x_{2}\right)$, number of added equipment $\left(x_{3}\right)$, labor hours last season $\left(x_{4}\right)$, rejection rate $\left(x_{5}\right)$, average number of processes $\left(x_{6}\right)$, and output last season $\left(x_{7}\right)$ as the evaluation parameters. Table 3 illustrates the relative importance ratio of these parameters.

We can get the judgment matrix $R$ based on the data in Table 3:

$$
R=\left[\begin{array}{ccccccc}
1 & 5 & 5 & 9 & 3 & 3 & 2 \\
\frac{1}{5} & 1 & \frac{1}{2} & 3 & 1 & \frac{1}{3} & \frac{1}{4} \\
\frac{1}{5} & 2 & 1 & 3 & \frac{1}{2} & \frac{1}{3} & \frac{1}{4} \\
\frac{1}{9} & \frac{1}{3} & \frac{1}{3} & 1 & \frac{1}{5} & \frac{1}{7} & \frac{1}{8} \\
\frac{1}{3} & 1 & 2 & 5 & 1 & 1 & 2 \\
\frac{1}{3} & 3 & 3 & 7 & \frac{1}{2} & 1 & 2 \\
\frac{1}{2} & 4 & 4 & 8 & \frac{1}{2} & \frac{1}{2} & 1
\end{array}\right] .
$$

The weight of every evaluation parameter can be calculated by the feature vector of $R$, as shown in Table 4 .

It can be found in Table 4 that $x_{2}, x_{3}$, and $x_{6}$ are the three lowest weight parameters, so they should be removed. Then we will obtain the dynamic-improved model based on the data in 1997-2007, 1998-2008, 1999-2009, 2000-2010, and 2001-2011 as follows. Equation (24) is also called Model B':

$$
\begin{aligned}
y_{2007}= & -10.50-0.0356 x_{1}+0.130 x_{4}+0.321 x_{5}+0.8503 x_{7}, \\
y_{2008}= & -10.44-0.041 x_{1}+0.121 x_{4}+0.342 x_{5}+0.84 x_{7}, \\
y_{2009}= & -10.57-0.043 x_{1}+0.119 x_{4}+0.355 x_{5}+0.82 x_{7}, \\
y_{2010}= & -10.6203-0.0325 x_{1}+0.118 x_{4}-0.325 x_{5} \\
& +0.875 x_{7}, \\
y_{2011}= & -10.43-0.0357 x_{1}+0.135 x_{4}-0.412 x_{5}+0.862 x_{7} .
\end{aligned}
$$

Now, the traditional model (Model A), the staticimproved model (Model B), and the dynamic-improved model (Model $\mathrm{B}^{\prime}$ ) have been obtained. Then our task is to determine which one is the best model.

\section{Evaluation of The Models and Error Analysis}

First, the DHNN evaluation method is adopted to estimate these three models. About 800 neurons are needed in the process of the estimation. 1 is lowest score, and 8 is the highest score. Table 5 shows these three models' scores of every estimation parameter; the scores are derived from the application evaluation of the models by the experts.

The neural network should be trained; then it will have 8 memory modes, and these are the 8 levels of the evaluation result. When the output of the network is stable, the status of the neurons in every level is shown in Table 6. After the training, we will obtain the evaluation result of these three models as given in Table 7 .

The dynamic-improved model (Model $\mathrm{B}^{\prime}$ ) has the highest level as shown in Table 7. So the output prediction which is gained from Model $\mathrm{B}^{\prime}$ should be the best.

Second, the Akaike information criterion (AIC) is adopted to measure which model is the best one. In the general case, the AIC is as follows:

$$
\mathrm{AIC}=2 k-2 \ln (\widehat{\theta}),
$$




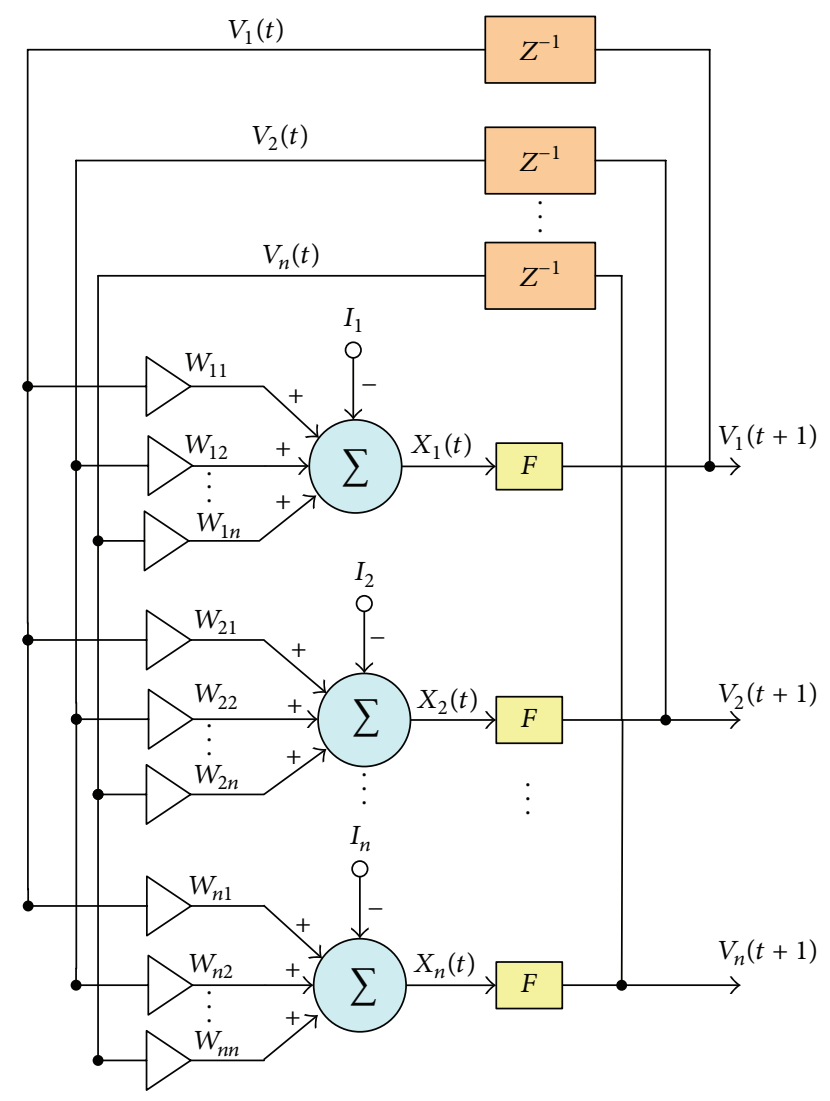

Figure 2: Structure of DHNN.

TABLE 7: Level result of every model.

\begin{tabular}{lccc}
\hline Models & Model A & Model B & Model B' $^{\prime}$ \\
\hline Level & 5 & 7 & 8 \\
\hline
\end{tabular}

TABLE 8: AIC value of every model.

\begin{tabular}{llcc}
\hline Models & $k$ & $\hat{\theta}$ & AIC value \\
\hline Model A & 7 & 6.14 & 10.37 \\
Model B & 4 & 2.38 & 6.27 \\
Model B & 4 & 2.44 & 6.22 \\
\hline
\end{tabular}

where $k$ is the number of parameters in the model and $\hat{\theta}$ is the maximized value of the likelihood function for the estimated model.

Let $x_{1}, x_{2}, \ldots, x_{n}$ be one value on the sample $X_{1}, X_{2}, \ldots, X_{n}$. Then the probability is as follows:

$$
L(\theta)=L\left(x_{1}, x_{2}, \ldots, x_{n} ; \theta\right)=\prod_{i=1}^{n} p\left(x_{i} ; \theta\right), \quad \theta \in \Theta .
$$

If $\widehat{\theta}$ exists, then

$$
L\left(x_{1}, x_{2}, \ldots, x_{n} ; \widehat{\theta}\right)=\max _{\theta \in \Theta} L\left(x_{1}, x_{2}, \ldots, x_{n} ; \theta\right),
$$

where $\widehat{\theta}$ is the maximized value of the likelihood function.

We apply (25), (26), and (27) to calculate the AIC value of every model as shown in Table 8.

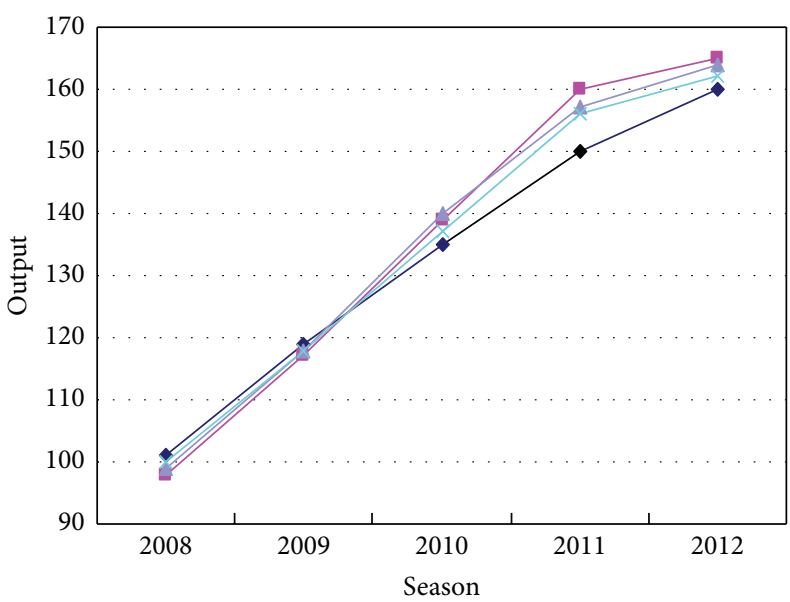

$\rightarrow$ Actual output $\rightarrow$ Output prediction of Model B
$\rightarrow$ Output prediction of Model A $\rightarrow$ Output prediction of Model B'

FIGURE 3: Line chart of output predictions and actual output.

From Table 8, we can find that Model $\mathrm{B}^{\prime}$ has the minimum AIC value. So Model B' (the dynamic-improved model) is the best one. This result is the same with the DHNN evaluation method.

To validate the analysis results of DHNN and AIC evaluation, we use these three models separately to gain the output prediction. The comparison of output predictions of these three models and actual output is illustrated in Table 9. We use mean absolute percentage error (MAPE) as follows to measure the errors of the models:

$$
\text { MAPE }=\left(\frac{100}{n}\right) \sum_{i=1}^{n}\left|\frac{A_{i}-F_{i}}{A_{i}}\right|,
$$

where $A_{i}$ is the actual value, $F_{i}$ is the prediction value, and $n$ is the number of error.

The line chart of the comparison is also given in Figure 3.

Figure 4 shows the error comparison of Model A, Model B, and Model B' from 2008 to 2012. Figure 4 shows the MAPE of every model in these five years.

It can be found in Table 8, Figures 3, 4, and 5 that if we use Model A to predict the output from 2008 to 2012, the maximum error is $-6.67 \%$ in 2011, the minimum error is $1.68 \%$ in 2009 , and the MAPE is $3.48 \%$.

If we use Model $\mathrm{B}$ to predict the output, the maximum error is $-4.67 \%$ in 2011 , the minimum error is $0.84 \%$ in 2009 , and the MAPE is $2.74 \%$. So Model B is better than Model A.

Finally, if we use the dynamic-improved model (Model $\left.\mathrm{B}^{\prime}\right)$ to calculate the output, the maximum error is $-4.00 \%$, the minimum error is $0.84 \%$, and the MAPE is $1.71 \%$. So, among these three models, Model B' is the best one. This comparison result is the same with the DHNN evaluation and AIC value on the three models. Therefore, Model $\mathrm{B}^{\prime}$ which is obtained by DHNN evaluation and AIC value has the practical value in output prediction. 
TABLE 9: Comparison of output predictions and actual output.

\begin{tabular}{|c|c|c|c|c|c|c|c|}
\hline Seasons & Actual output & $\begin{array}{c}\text { Output } \\
\text { prediction of } \\
\text { Model A }\end{array}$ & $\begin{array}{c}\text { Output } \\
\text { prediction of } \\
\text { Model B }\end{array}$ & $\begin{array}{c}\text { Output } \\
\text { prediction of } \\
\text { Model B' }\end{array}$ & $\begin{array}{l}\text { Error of } \\
\text { Model A }\end{array}$ & $\begin{array}{l}\text { Error of } \\
\text { Model B }\end{array}$ & $\begin{array}{c}\text { Error of } \\
\text { Model B' }\end{array}$ \\
\hline 2008 & 101 & 98 & 99 & 100 & $2.97 \%$ & $1.98 \%$ & $0.99 \%$ \\
\hline 2009 & 119 & 117 & 118 & 118 & $1.68 \%$ & $0.84 \%$ & $0.84 \%$ \\
\hline 2010 & 135 & 139 & 140 & 137 & $-2.96 \%$ & $-3.70 \%$ & $-1.48 \%$ \\
\hline 2011 & 150 & 160 & 157 & 156 & $-6.67 \%$ & $-4.67 \%$ & $-4.00 \%$ \\
\hline 2012 & 160 & 165 & 164 & 162 & $-3.13 \%$ & $-2.50 \%$ & $-1.25 \%$ \\
\hline \multicolumn{5}{|c|}{ Mean absolute percentage error (MAPE) } & $3.48 \%$ & $2.74 \%$ & $1.71 \%$ \\
\hline
\end{tabular}

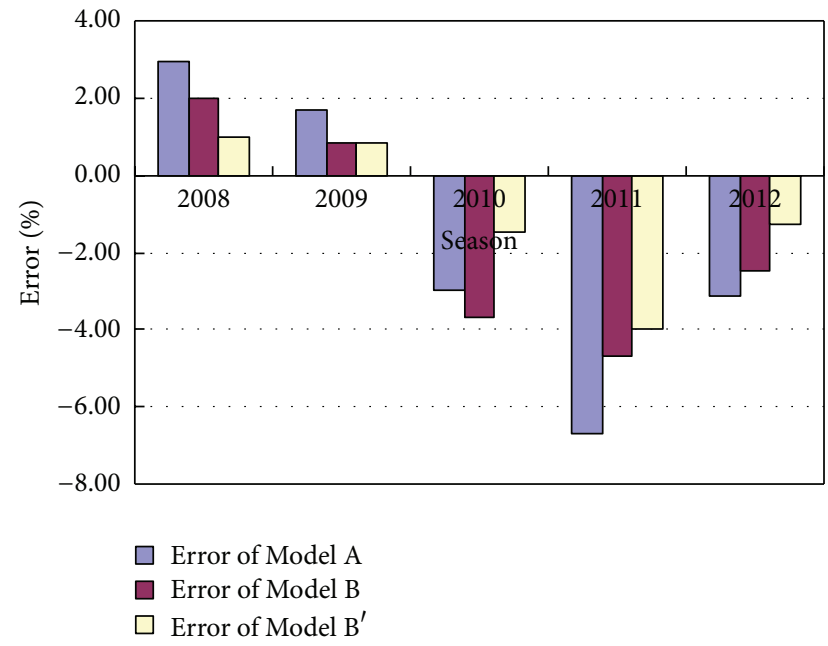

FIgURE 4: Error comparison of Model A, Model B, and Model B'.

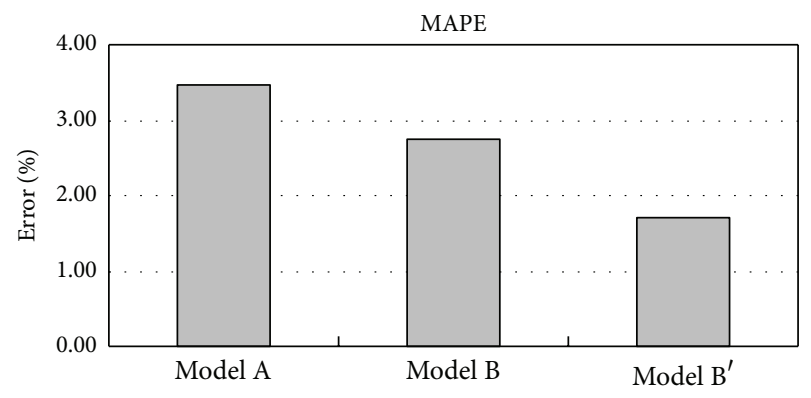

Figure 5: MAPE of Model A, Model B, and Model B'.

\section{Conclusions}

The output prediction is an essential part of manufacturing production management system. In this paper, a dynamicimproved multiple linear regression model based on parameter evaluation using DHNN was presented. Then based on the production data of an aviation enterprise from 1996 to 2012, the traditional model, static-improved model, and dynamicimproved model based on DHNN evaluation and AIC value were established. The results of output prediction from 2008 to 2012 were obtained using these three models; compared with the actual output, we got the discrepancies of the models, and the model based on DHNN evaluation and AIC value had the minimum discrepancy. So, the model based on DHNN evaluation and AIC value has a good practical value in output prediction of production management.

\section{Conflict of Interests}

The authors declare that there is no conflict of interests regarding the publication of this paper.

\section{Acknowledgments}

This work was supported by the Fundamental Research Funds for the Central Universities under Grant no. K5051204001 and Shaanxi Provincial Scientific and Technological Research Projects under Grant no. DF0102130401. The authors would like to appreciate the editor, associate editor, and the reviewers for their valuable comments and suggestions.

\section{References}

[1] H. T. Wagner, S. C. Morton, A. R. J. Dainty, and N. D. Burns, "Path dependent constraints on innovation programmes in production and operations management," International Journal of Production Research, vol. 49, no. 11, pp. 3069-3085, 2011.

[2] W.-H. Hung, C.-F. Ho, J.-J. Jou, and K.-H. Kung, "Relationship bonding for a better knowledge transfer climate: an ERP implementation research," Decision Support Systems, vol. 52, no. 2, pp. 406-414, 2012.

[3] C. J. Ho and T. C. Ireland, "Mitigating forecast errors by lot-sizing rules in ERP-controlled manufacturing systems," International Journal of Production Research, vol. 50, no. 11, pp. 3080-3094, 2012.

[4] S. C. L. Koh, A. Gunasekaran, and S. M. Saad, "Tackling uncertainty in ERP-controlled manufacturing environment: a knowledge management approach," International Journal of Advanced Manufacturing Technology, vol. 31, no. 7-8, pp. 833840, 2007.

[5] N. M. Matsveichuk, Yu. N. Sotskov, N. G. Egorova, and T.-C. Lai, "Schedule execution for two-machine flow-shop with interval processing times," Mathematical and Computer Modelling, vol. 49, no. 5-6, pp. 991-1011, 2009.

[6] C. T. Ng, N. M. Matsveichuk, Y. N. Sotskov, and T. C. E. Cheng, "Two-machine flow-shop minimum-length scheduling with 
interval processing times," Asia-Pacific Journal of Operational Research, vol. 26, no. 6, pp. 715-734, 2009.

[7] N. M. Matsveichuk, Y. N. Sotskov, and F. Werner, "The dominance digraph as a solution to the two-machine flow-shop problem with interval processing times," Optimization, vol. 60, no. 12, pp. 1493-1517, 2011.

[8] R. Y. Zhong, Q. Y. Dai, T. Qu, G. J. Hu, and G. Q. Huang, "RFIDenabled real-time manufacturing execution system for masscustomization production," Robotics and Computer-Integrated Manufacturing, vol. 29, no. 2, pp. 283-292, 2013.

[9] S. F. Huin, L. H. S. Luong, and K. Abhary, "Knowledge-based tool for planning of enterprise resources in ASEAN SMEs," Robotics and Computer-Integrated Manufacturing, vol. 19, no. 5, pp. 409-414, 2003.

[10] A. Amirteimoori and S. Kordrostami, "Production planning: a DEA-based approach," International Journal of Advanced Manufacturing Technology, vol. 56, no. 1-4, pp. 369-376, 2011.

[11] B. Golany, "An interactive MOLP procedure for the extension of DEA to effectiveness analysis," Journal of the Operational Research Society, vol. 39, no. 8, pp. 725-734, 1988.

[12] J. Du, L. Liang, Y. Chen, and G.-B. Bi, "DEA-based production planning," Omega, vol. 38, no. 1-2, pp. 105-112, 2010.

[13] P. Korhonen and M. Syrjänen, "Resource allocation based on efficiency analysis," Management Science, vol. 50, no. 8, pp. 11341144, 2004.

[14] H. Kim, H.-I. Jeong, and J. Park, "Integrated model for production planning and scheduling in a supply chain using benchmarked genetic algorithm," International Journal of Advanced Manufacturing Technology, vol. 39, no. 11-12, pp. 1207-1226, 2008.

[15] J. B. Lasserre, "An integrated model for job-shop planning and scheduling," Management Science, vol. 38, no. 8, pp. 1201-1211, 1992.

[16] S. Dauzère-Péres and J.-B. Lasserre, "Integration of lotsizing and scheduling decisions in a job-shop," European Journal of Operational Research, vol. 75, no. 2, pp. 413-426, 1994.

[17] M. F. Anwar and R. Nagi, "Integrated lot-sizing and scheduling for just-in-time production of complex assemblies with finite set-ups," International Journal of Production Research, vol. 35, no. 5, pp. 1447-1470, 1997.

[18] G. Xiong and T. R. Nyberg, "Push/pull production plan and schedule used in modern refinery CIMS," Robotics and Computer-Integrated Manufacturing, vol. 16, no. 6, pp. 397-410, 2000.

[19] X.-D. Zhang and H.-S. Yan, "Integrated optimization of production planning and scheduling for a kind of job-shop," International Journal of Advanced Manufacturing Technology, vol. 26, no. 7-8, pp. 876-886, 2005.

[20] G. Fandel and C. Stammen-Hegene, "Simultaneous lot sizing and scheduling for multi-product multi-level production," International Journal of Production Economics, vol. 104, no. 2, pp. 308-316, 2006.

[21] M. Chazal, E. Jouini, and R. Tahraoui, "Production planning and inventories optimization: a backward approach in the convex storage cost case," Journal of Mathematical Economics, vol. 44, no. 9-10, pp. 997-1023, 2008.

[22] Y. Pochet and L. A. Wolsey, Production Planning by Mixed Integer Programming, Springer Series in Operations Research and Financial Engineering, Springer, New York, NY, USA, 2006.

[23] P. Damodaran and M. C. Vélez-Gallego, "A simulated annealing algorithm to minimize makespan of parallel batch processing machines with unequal job ready times," Expert Systems with Applications, vol. 39, no. 1, pp. 1451-1458, 2012.

[24] E. Kaslik and S. Balint, "Complex and chaotic dynamics in a discrete-time-delayed Hopfield neural network with ring architecture," Neural Networks, vol. 22, no. 10, pp. 1411-1418, 2009. 


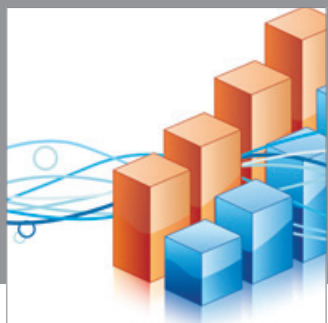

Advances in

Operations Research

mansans

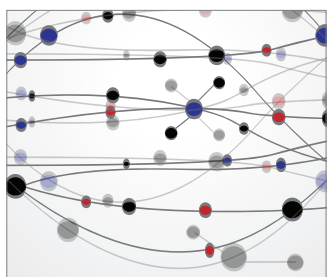

The Scientific World Journal
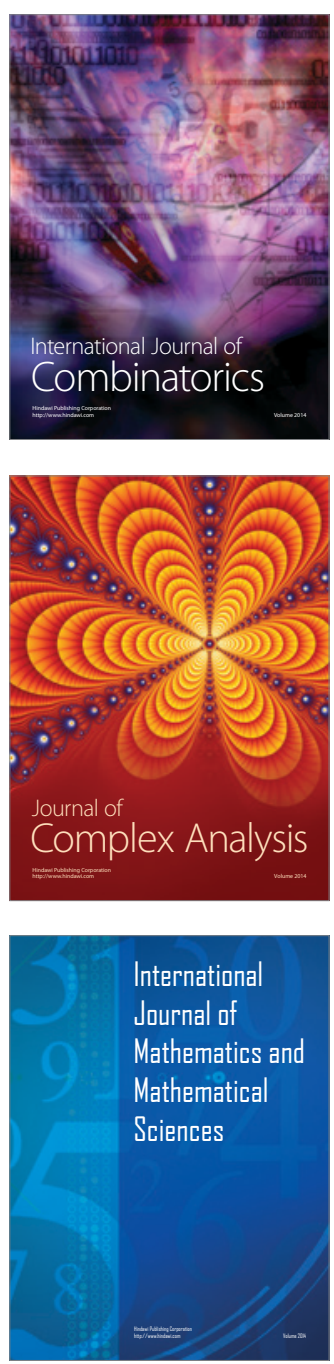
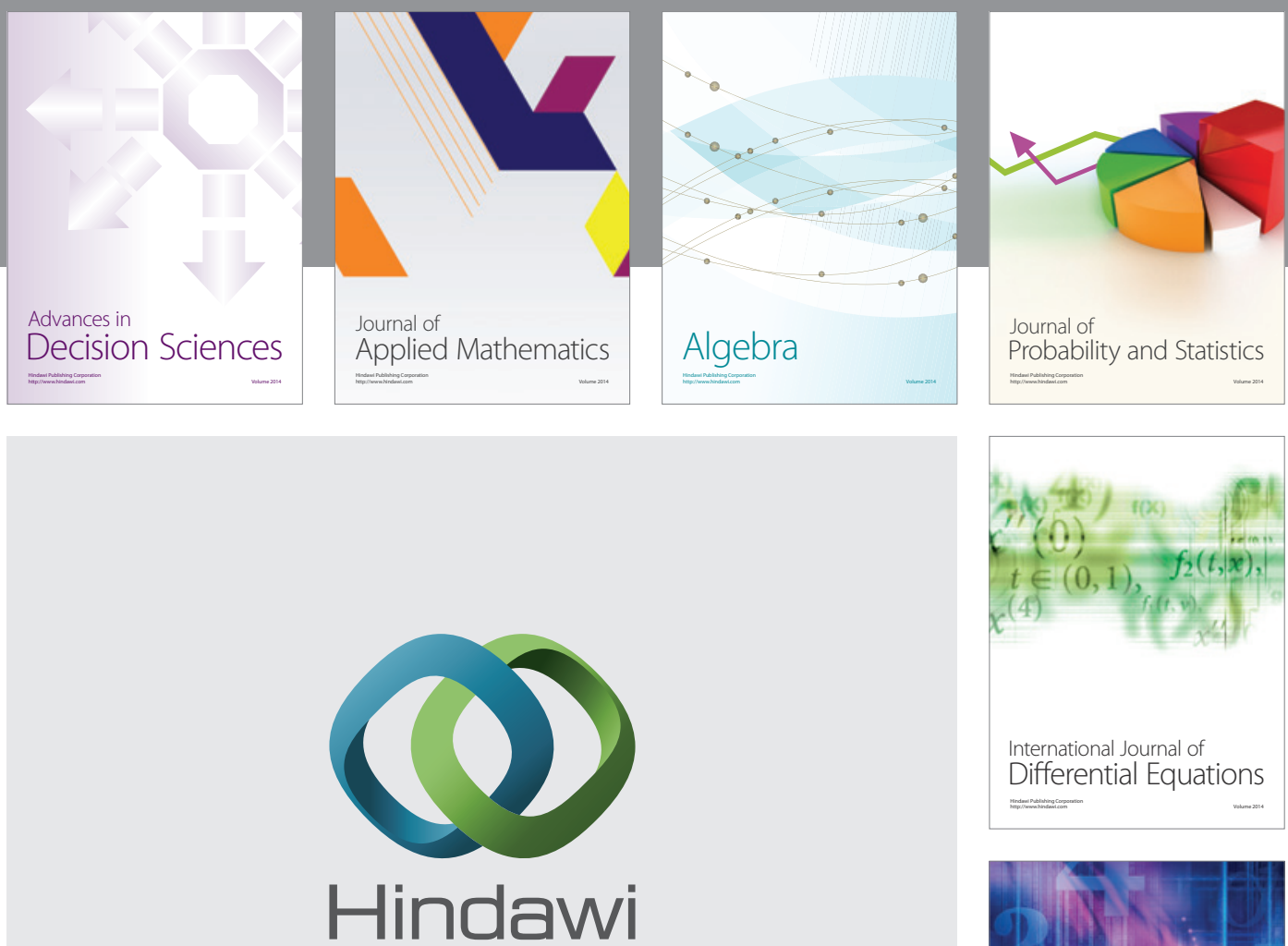

Submit your manuscripts at http://www.hindawi.com
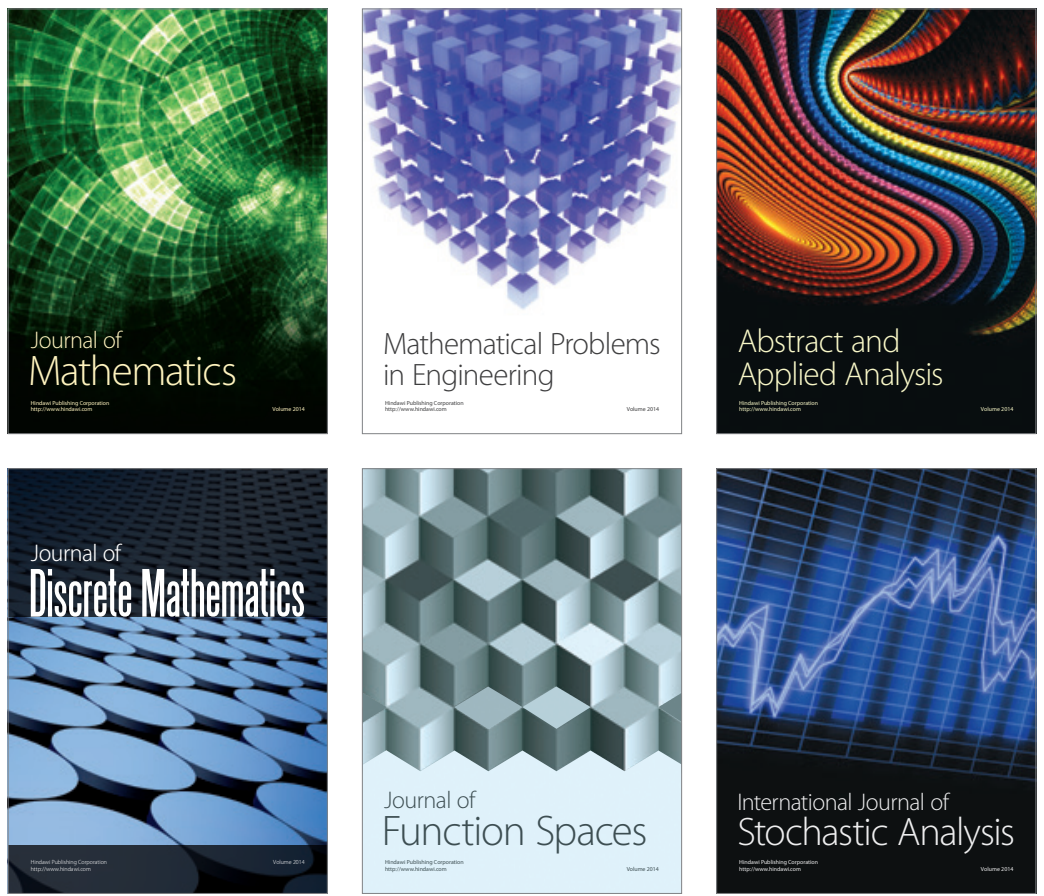

Journal of

Function Spaces

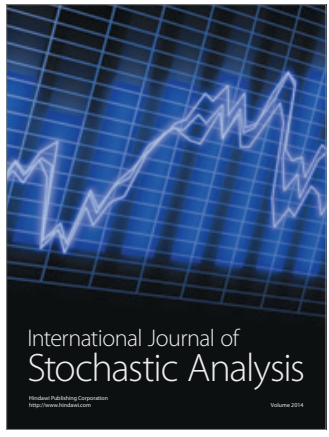

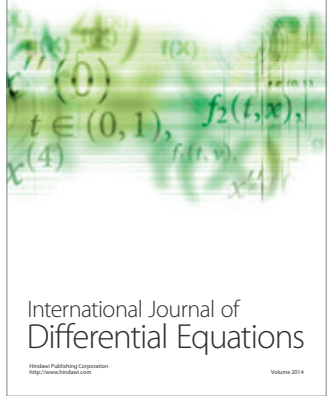
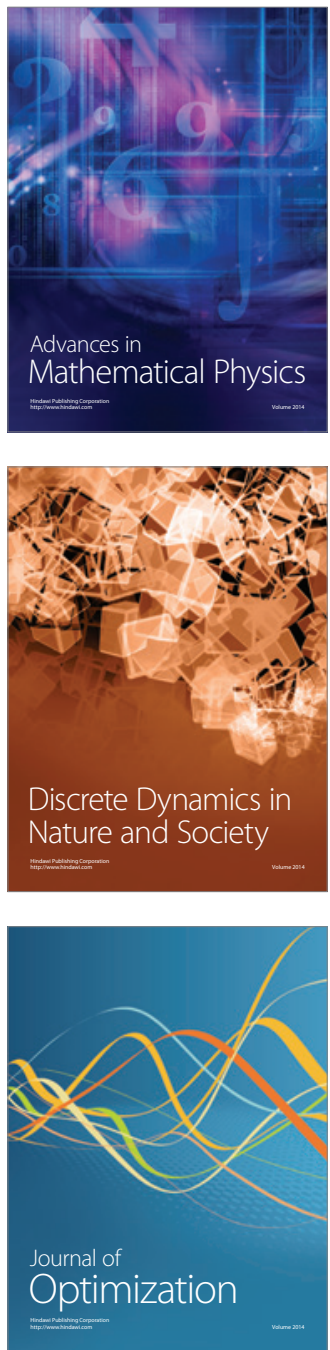